\title{
Fitting the Lattice Vacuum Polarisation Function to Perturbation Theory
}

\author{
Gregorio Herdoíza $^{* a}$, Hanno Horch ${ }^{a}$, Benjamin Jäger ${ }^{a, b}$, Hartmut Wittig ${ }^{a, b}$ \\ ${ }^{a}$ PRISMA Cluster of Excellence, Institut für Kernphysik \\ Johannes Gutenberg-Universität, 55099 Mainz, Germany \\ ${ }^{b}$ Helmholtz Institute Mainz \\ Johannes Gutenberg-Universität, 55099 Mainz, Germany \\ E-mail: \{herdoiza, horch, jaeger,wittig\} akph.uni-mainz.de
}

\begin{abstract}
The hadronic vacuum polarisation function computed with two flavours of $\mathrm{O}(a)$ improved Wilson fermions is studied in the large momentum region. Three values of the lattice spacing are used to monitor the size of discretisation effects. A comparison to perturbative QCD expressions including the dimension-four contributions from the Operator Product Expansion is performed. The systematic effects present in this matching to perturbation theory are explored.
\end{abstract}

31st International Symposium on Lattice Field Theory - LATTICE 2013

July 29 - August 3, 2013

Mainz, Germany

\footnotetext{
*Speaker.
} 


\section{Introduction}

The QCD contributions to the photon vacuum polarisation function (VPF), $\Pi\left(q^{2}\right)$, are currently responsible for a large fraction of the theoretical uncertainties in the determination of the anomalous magnetic moment of the muon $a_{\mu}$ or of the running of the QED coupling constant $\alpha_{\mathrm{QED}}$. The VPF depends on the squared momentum transfer $q^{2}$. In the space-like (Euclidean) region, the large $q^{2}$ regime of the VPF can be suitably described by perturbative QCD (pQCD) due to the properties of asymptotic freedom and the absence of resonance effects. On the other hand, the low-energy structure of QCD, where confinement and the spontaneous breaking of chiral symmetry are prominent, is beyond the domain of validity of pQCD. These non-perturbative strong interaction effects are responsible for the large uncertainties in the hadronic contribution to $a_{\mu}$.

In a study of the $q^{2}$-dependence of the VPF, the question of the regime of validity of the pQCD expansion in powers of strong coupling $\alpha_{s}$ needs to be addressed. An additional ingredient can be considered in this analysis, by performing an operator product expansion (OPE) of the two currents defining the vacuum polarisation tensor $\Pi_{\mu v}(q)$. In this way, non-perturbative effects can be incorporated through terms formally organised in an expansion in powers of $1 / q^{2}$ up to logarithms. In the OPE, the operator matrix elements will capture the long-range strong interaction properties while the perturbative Wilson coefficients will encode the short distance physics.

Lattice QCD provides a robust method to study the $q^{2}$-dependence of the VPF and of its logarithmic derivative which is related to the Adler function. In this way, both the low-energy regime that is essential for current phenomenological studies, and a region of larger $q^{2}$ values can be studied from first principles. If this high-energy regime is sufficiently large such that contact with pQCD can be safely made, then the lattice measurement of $\Pi\left(q^{2}\right)$ provides a way to determine the coupling $\alpha_{s}$ and to derive the scale parameter $\Lambda_{\mathrm{QCD}}$.

The matching of a lattice determination of $\Pi\left(q^{2}\right)$ to its OPE counterpart should thus be done at sufficiently large $q^{2}$ values in order to guarantee the good convergence properties of the perturbative expansion. However, lattice discretisation effects increase with $q^{2}$ and should be considered under control provided that the momentum transfer remains much smaller than the cutoff, i.e. $(a q)^{2} \ll 1$. Since the typical values of the lattice spacing $a$ of current simulations are in a region, $a^{-1} \in[2,4] \mathrm{GeV}$, the $q^{2}$ values should satisfy $q^{2} \ll 16 \mathrm{GeV}^{2}$ for the finest values of $a$ and even stronger constraints for coarser lattice spacings. Assuming that these constraints allow to identify a region of $q^{2}$ where the lattice VPF can be matched to the OPE, it is still important that this region is sufficiently large in order to probe the expected logarithmic dependence in $q^{2}$ of the VPF.

The lattice group in Mainz has a dedicated research program [1] aiming at a precise determination of physical observables related to the VPF. In this study, we explore the possibility of performing such a matching of the lattice VPF to $\mathrm{pQCD}$ in order to determine $\Lambda_{\mathrm{QCD}}$. Two ingredients are particularly relevant for this analysis: (i) the existence of statistically accurate lattice data for the VPF over a relatively large interval of $q^{2}$, with sufficient values of the lattice spacing in order to monitor the size of discretisation effects and (ii) the availability of pQCD results to high-order in the $\alpha_{s}$ expansion. 


\section{The Vacuum Polarisation Function and its Operator Product Expansion}

The Euclidean hadronic vacuum polarisation tensor is defined as,

$$
\Pi_{\mu v}(q)=\int d^{4} x e^{i q x}\left\langle J_{\mu}(x) J_{v}(0)\right\rangle,
$$

where the vector current reads,

$$
J_{\mu}(x)=\sum_{f=1}^{N_{\mathrm{f}}} Q_{f} \bar{\psi}_{f}(x) \gamma_{\mu} \psi_{f}(x)
$$

We consider the $N_{\mathrm{f}}=2$ theory for which $\psi_{f}(x)=\left(\psi_{u}, \psi_{d}\right)$ is made of mass-degenerate quark fields with electric charges $Q_{f}=(2 / 3,-1 / 3)$. Euclidean invariance and current conservation lead to,

$$
\Pi_{\mu v}(q)=\left(g_{\mu v} q^{2}-q_{\mu} q_{v}\right) \Pi\left(q^{2}\right)
$$

The VPF $\Pi\left(q^{2}\right)$ can be decomposed into non-singlet and singlet contributions. In the following, only quark-connected contributions to the VPF will be considered both on the lattice and in perturbation theory. For large space-like momenta, the non-singlet contribution to the VPF can be expressed in the OPE in the following way,

$$
\begin{aligned}
\Pi_{\mathrm{OPE}}\left(\alpha_{s}, q^{2}, m_{f}\right) & =\frac{5}{9}\left\{c+c_{0}\left(\alpha_{s}, \mu^{2}, q^{2}\right)+c_{m}\left(\alpha_{s}, \mu^{2}, q^{2}\right) \frac{\left(\bar{m}_{f}(q)\right)^{2}}{q^{2}}\right. \\
& \left.+c_{\Sigma}\left(\alpha_{s}, \mu^{2}, q^{2}\right) \frac{m_{f}\left\langle\bar{\psi}_{f} \psi_{f}\right\rangle}{q^{4}}+c_{4}\left(\alpha_{s}, \mu^{2}, q^{2}\right) \frac{\left\langle O_{\mathrm{OPE}}^{(4)}\right\rangle}{q^{4}}\right\}+O\left(\frac{1}{q^{6}}\right)
\end{aligned}
$$

The constant $c$ in the first term is scheme-dependent and diverges in the limit of infinite cutoff. The Wilson coefficients $c_{0}, c_{m}, c_{\Sigma}$ and $c_{4}$ in eq. (2.4) can be computed in perturbation theory and, depending on the case, they are known from 1- to 4-loop order in the $\alpha_{s}$ expansion [3-5]. The non-perturbative physics is encoded in the condensates $\left\langle\bar{\psi}_{f} \psi_{f}\right\rangle,\left\langle O_{\mathrm{OPE}}^{(4)}\right\rangle, \ldots$

The perturbative expressions are defined in the $\overline{\mathrm{MS}}$ scheme. The connection of the coupling $\alpha_{s}$ to the scale parameter $\Lambda_{\overline{M S}}^{\left(N_{\mathrm{f}}=2\right)}$ is given by the 4-loop $\beta$-function [6]. The renormalised quark mass $\bar{m}_{f}(\mu)$ refers to the quark mass at the renormalisation scale $\mu[2,7]$. For a physical quantity such as the Adler function, $D\left(q^{2}\right)=-12 \pi^{2} d \Pi\left(q^{2}\right) / d \log \left(q^{2}\right)$, the contribution from the constant $c$ in eq. (2.4) vanishes and the contributions from other terms remain finite. Moreover, any scheme and scale dependence should disappear as higher order terms are included.

\section{Lattice Determination of the VPF and OPE fits}

The lattice action is made of two flavours of non-perturbatively $\mathrm{O}(a)$ improved Wilson fermions and the Wilson plaquette gauge action. We use a lattice version of the vacuum polarisation tensor in eq. (2.1) where the local current, $J_{\mu}^{(l)}(x)=Z_{\mathrm{V}} \bar{\psi}_{f}(x) \gamma_{\mu} \psi_{f}(x)$, is inserted at the source and the point-split conserved current $J_{\mu}^{(\mathrm{p} . \mathrm{s})}(x)$ at the sink. The lattice momentum is given by $\hat{q}_{\mu}=2 / a \sin \left(a q_{\mu} / 2\right)$, where $q_{\mu}=2 \pi n_{\mu} / L$ and $n_{\mu} \in[0,1, \ldots, L / a-1]$. 


\begin{tabular}{cccccccc}
\hline$\beta$ & $a[\mathrm{fm}]$ & lattice & $L[\mathrm{fm}]$ & $m_{\pi}[\mathrm{MeV}]$ & $m_{\pi} L$ & $|a \hat{q}|_{\max }$ & Ens. \\
\hline 5.20 & 0.079 & $64 \times 32^{3}$ & 2.5 & 312 & 4.0 & 1.05 & A5 \\
\hline 5.30 & 0.063 & $64 \times 32^{3}$ & 2.0 & 451 & 4.7 & 1.05 & E5 \\
& & $96 \times 48^{3}$ & 3.0 & 324 & 5.0 & & F6 \\
\hline 5.50 & 0.050 & $96 \times 48^{3}$ & 2.4 & 430 & 5.2 & 0.93 & N5 \\
& & & & 330 & 4.1 & & N6 \\
& & $128 \times 64^{3}$ & 3.2 & 260 & 4.4 & & O7 \\
\hline
\end{tabular}

Table 1: Ensembles - generated by the CLS initiative - with two flavours of $\mathrm{O}(a)$ improved Wilson fermions used in this study.

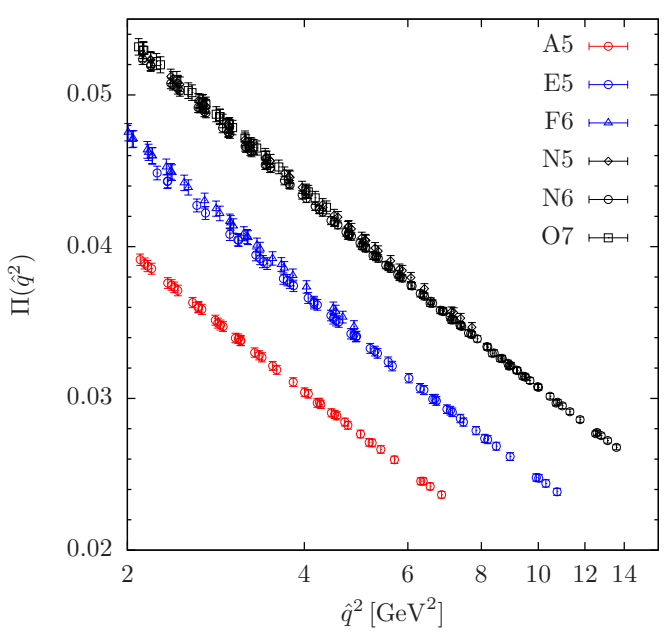

(a)

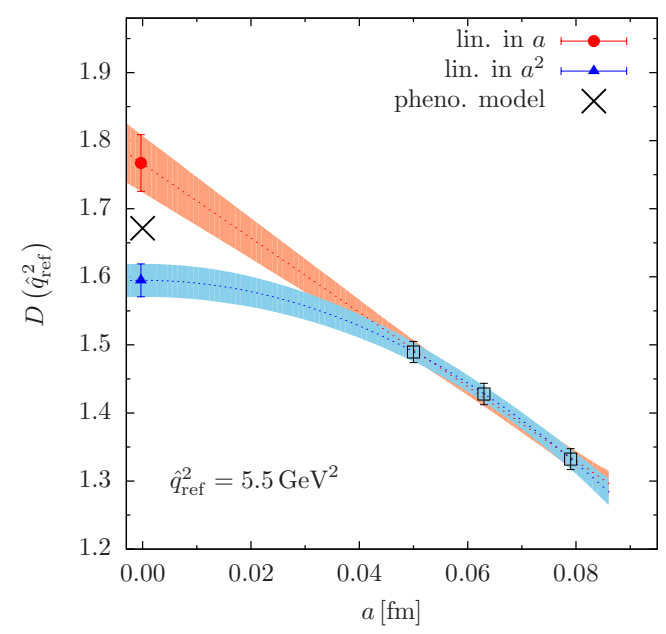

(b)

Figure 1: (a) The lattice $\operatorname{VPF} \Pi\left(\hat{q}^{2}\right)$ as determined from the ensembles in table 1. (b) Continuum-limit scaling analysis of the Adler function, $D\left(\hat{q}_{\text {ref }}^{2}\right)$, at a reference momentum transfer $\hat{q}_{\text {ref }}^{2}=5.5 \mathrm{GeV}^{2}$.

The ensembles used in this study are listed in table 1 . Three values of the lattice spacing including ensembles with a cutoff $a^{-1} \approx 4 \mathrm{GeV}$ - are used to monitor the size of discretisation effects. The maximal values of the momentum $|a \hat{q}|_{\max }$ at which the VPF has been computed are also reported table 1 . The $\hat{q}^{2}$ range where the VPF has been determined is also illustrated in fig. 1(a) where the vertical displacement among data from different lattice spacings mainly arises from the unphysical constant $c$ in eq. (2.4). The comparison of ensembles with different quark masses indicates that, given the current statistical precision on the VPF (below 1\%), mass effects are rather small for $\hat{q}^{2}>4 \mathrm{GeV}^{2}$.

The Adler function $D\left(\hat{q}^{2}\right)$ is a convenient observable to monitor cutoff effects in the $\hat{q}^{2}$ dependence of the VPF. Indeed, $D\left(\hat{q}^{2}\right)$ is dimensionless and, as already mentioned, the contribution from unphysical constant $c$ in eq. (2.4) vanishes. We perform a continuum limit scaling analysis of the Adler function at a reference momentum transfer, $\hat{q}_{\text {ref }}^{2}=5.5 \mathrm{GeV}^{2}$. At this rather large value of $\hat{q}^{2}$, non-negligible contributions from cutoff effects can be anticipated. Since the VPF is derived from an off-shell matrix element, we expect that $\mathrm{O}(a)$ discretisation effects can be present in $D\left(\hat{q}_{\text {ref }}^{2}\right)$. In this preliminary study, the pion masses were not kept fixed to a reference value when approaching 


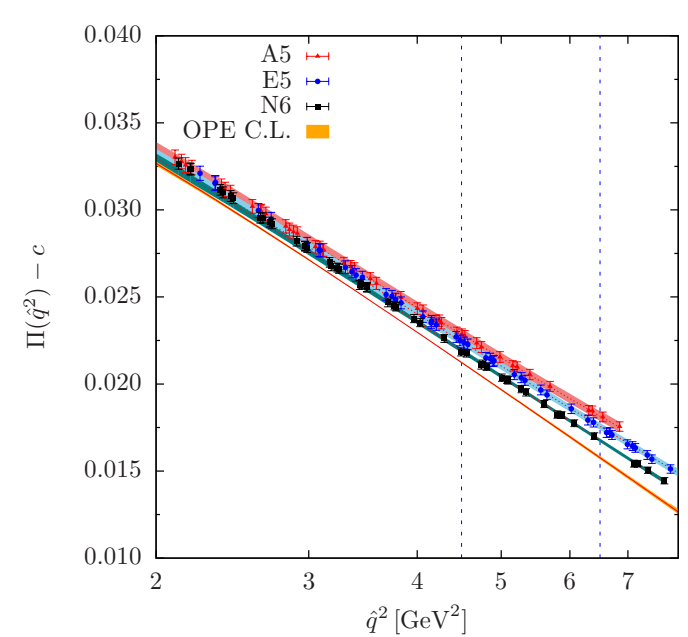

(a)

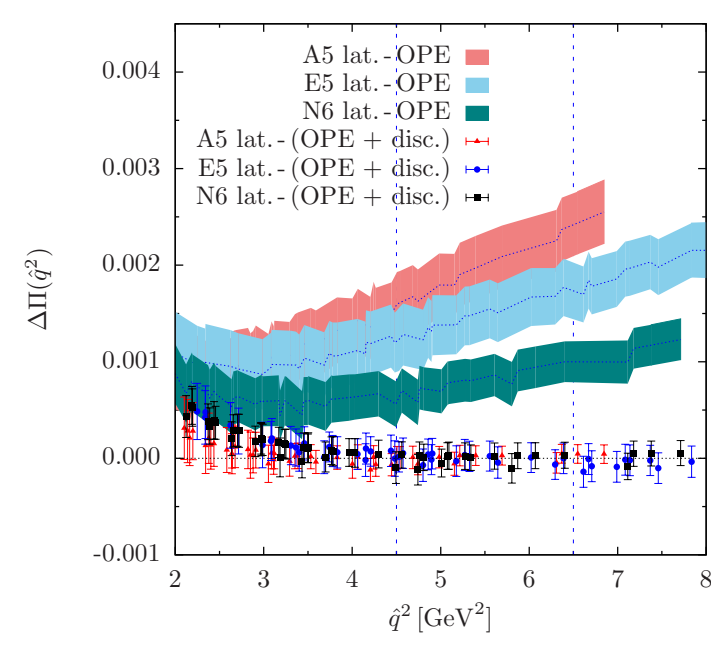

(b)

Figure 2: Illustration of a fit to the OPE expression in eq. (2.4) supplemented with terms of $\mathrm{O}\left((a \hat{q})^{2}\right)$ and $\mathrm{O}\left(a^{2} \log \left(\hat{q}^{2}\right)\right)$ to parametrise the lattice artefacts. Ensembles - N6, E5 and A5 in table 1 - with three different values of the lattice spacing were considered in the combined fit. The fit parameters $-\Lambda_{\overline{M S}}^{\left(N_{\mathrm{f}}=2\right)},\left\langle O_{\mathrm{OPE}}^{(4)}\right\rangle, c[\beta]$ and the two parameters related to $\hat{q}^{2}$-dependent lattice artefacts - were fitted in the interval of $\hat{q}^{2}$ indicated by the vertical dashed lines. The left panel shows the VPF after subtraction of the constant terms $c$. The result of the combined fit is shown by the coloured bands. The band labelled by "OPE C.L." refers to the continuum limit curve derived from the fit. The right panel shows the difference, $\Delta \Pi=\Pi_{\text {lat. }}-\Pi_{\text {fit }}$, of the lattice data and the fit result. The data points refer to the case where $\Pi_{\text {fit }}$ involves the complete fit ansatz where discretisation effects are included. The difference between the data points and the coloured bands denotes the size of $\hat{q}^{2}$-dependent lattice artefacts.

the continuum limit. However, we stress that a weak quark-mass dependence is observed in this high-energy regime (see fig. 1(a)).

The continuum limit scaling of $D\left(\hat{q}_{\text {ref }}^{2}\right)$ is shown in fig. 1(b). Our data from three values of the lattice spacing is not accurate enough to clearly discriminate among $\mathrm{O}(a)$ and $\mathrm{O}\left(a^{2}\right)$ lattice artefacts. The estimate of $D\left(\hat{q}_{\text {ref }}^{2}\right)$ from a phenomenological model [12] is shown as a qualitative comparison (we recall that the continuum limit scaling has currently been performed at unphysical pion masses). Further investigations of the Adler function in this lattice setup [13, 14] and of lattice artefacts in the Ward identity of the vacuum polarisation tensor [13] were reported at this conference. As expected, discretisation effects in the Adler function are observed [13] to diminish for smaller values of $\hat{q}^{2}$.

In matching the lattice VPF to its OPE expression in eq. (2.4) we augment the fit ansatz to include two terms parametrising discretisation effects of $\mathrm{O}\left((a \hat{q})^{2}\right)$ and $\mathrm{O}\left(a^{2} \log \left(\hat{q}^{2}\right)\right)$. With such an expression we perform a fit combining the VPF data from three values of $\beta$. The fit parameters are $\Lambda_{\overline{M S}}^{\left(N_{\mathrm{f}}=2\right)},\left\langle O_{\mathrm{OPE}}^{(4)}\right\rangle, c[\beta]$ and the two parameters related to $\hat{q}^{2}$-dependent lattice artefacts. In defining the RGI product, $m_{f}\left\langle\bar{\psi}_{f} \psi_{f}\right\rangle$, we use as input the value of the chiral condensate, $\left\langle\bar{\psi}_{u} \psi_{u}\right\rangle=-\left(0.270(7) \mathrm{GeV}^{3}\right)$, reported by FLAG [11]. Uncorrelated fits with loose priors on $c[\beta]$ and $\left\langle O_{\mathrm{OPE}}^{(4)}\right\rangle$ are considered. We refer to refs. [8-10] for earlier studies of the matching of the lattice VPF to the OPE.

An example of a combined fit of the lattice VPF from ensembles N6, E5 and A5 (see table 1) is 

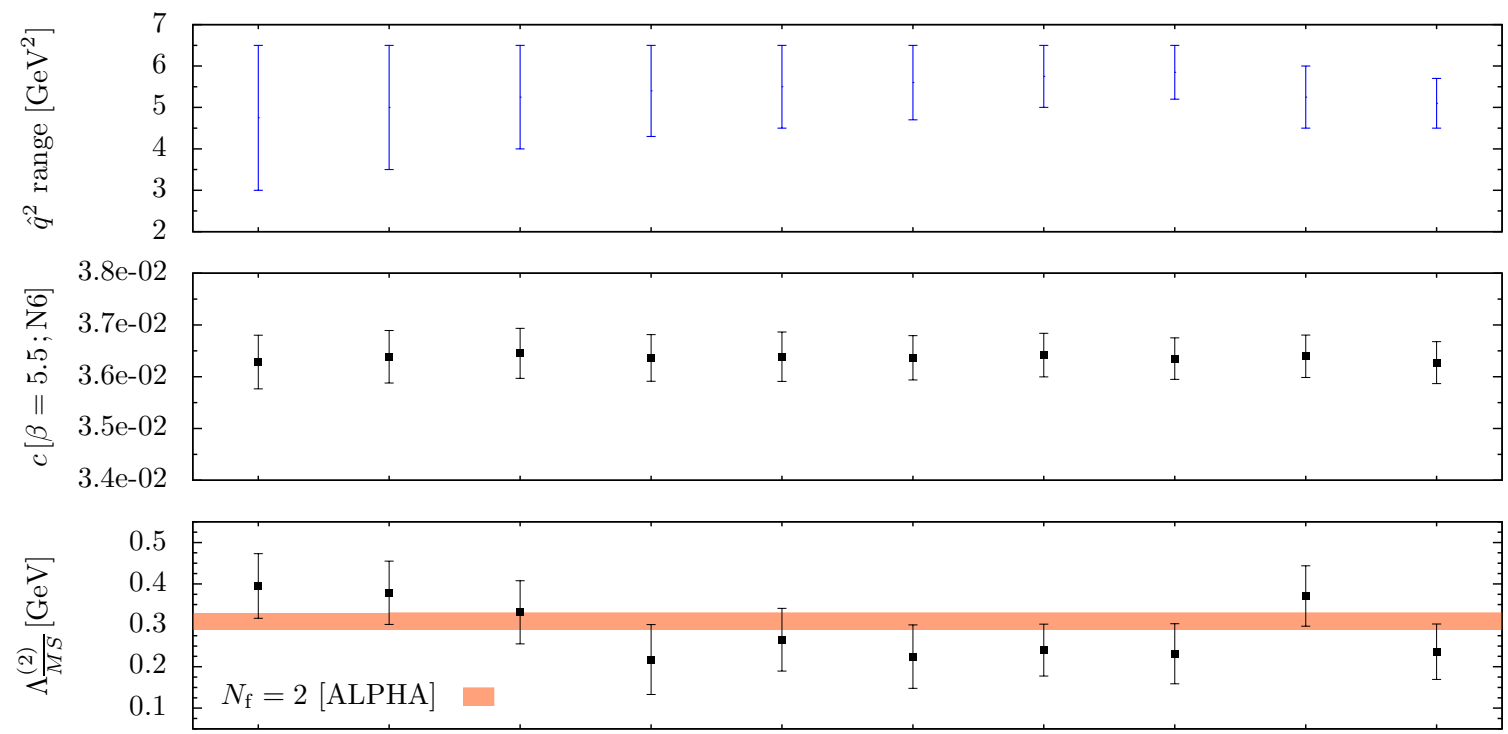

Figure 3: Comparison of combined fits of the lattice VPF to the OPE expression in eq. (2.4) - supplemented with terms of $\mathrm{O}\left((a \hat{q})^{2}\right)$ and $\mathrm{O}\left(a^{2} \log \left(\hat{q}^{2}\right)\right)$ - for different choices of the $\hat{q}^{2}$ range employed in the fit. The ensembles N6, E5 and A5, with lattices spacings ranging from $a \approx 0.05$ to $0.09 \mathrm{fm}$, were considered. In the upper panel, the vertical size of the bars indicates the $\hat{q}^{2}$ interval used in the fit. The middle and lower panels display the corresponding values of the fit parameters, $c[\beta=5.5]$ and $\Lambda_{\overline{M S}}^{\left(N_{\mathrm{f}}=2\right)}$, respectively. For the latter case, the $N_{\mathrm{f}}=2$ result [2] from the ALPHA collaboration, $\Lambda_{\overline{M S}}^{\left(N_{\mathrm{f}}=2\right)}=310(20) \mathrm{MeV}$, is indicated by the horizontal band.

shown in fig. 2. The fit uses the OPE expression in eq. (2.4) supplemented with terms parametrising lattice artefacts. Concerning $\hat{q}^{2}$-dependent cutoff effects, fig. 2 confirms the expectations based on the continuum-limit scaling of the Adler function. Indeed, a proper analysis of lattice artefacts is particularly important in the large $\hat{q}^{2}$ regime. We note in passing that results similar to those in fig. 2 are found in a fit where $\Lambda_{\overline{M S}}^{\left(N_{\mathrm{f}}=2\right)}$ is fixed to $310(20) \mathrm{MeV}$ [2].

The dependence of the fit parameters on the choice of the $\hat{q}^{2}$ interval is illustrated in fig. 3 . While the constants $c[\beta]$ appear to be fairly insensitive to the fit interval, we observe that the central value of $\Lambda \frac{\left(N_{\mathrm{f}}=2\right)}{M S}$ does depend on the $\hat{q}^{2}$ range although within rather large uncertainties. On the other hand, given the current accuracy, we do not observe a significant dependence of $\Lambda \frac{(2)}{M S}$ on the light-quark mass.

Further studies will be carried out to properly address the systematic effects present in this analysis. In particular, we will consider variations on the terms describing the lattice artefacts and their interplay with the other fit parameters.

\section{Conclusions}

We have performed a preliminary study of the matching of the lattice VPF to the OPE expression up to order $1 / q^{4}$. In the large $q^{2}$ regime where the perturbative expansion is valid, the lattice data tends to suffer from large discretisation effects. Fits including terms parametrising the $\hat{q}^{2}$-dependent lattice artefacts confirm the necessity of a proper analysis of the approach to the continuum limit when considering lattice spacings in the region, $a \approx 0.05$ to $0.09 \mathrm{fm}$. We observe a 
dependence of the extracted values of the $\Lambda \frac{\left(N_{\mathrm{f}}=2\right)}{M S}$ parameter on the choice of the $q^{2}$-interval considered in the fits. However, the statistical uncertainty on the extracted values of $\Lambda_{\overline{M S}}^{\left(N_{\mathrm{f}}=2\right)}$ also tends to be rather large.

As an extension to this work, we plan to subtract from the lattice data the leading discretisation effects computed in lattice perturbation theory. Moreover, variations of the combined fits regarding the treatment of the lattice spacing and quark mass dependence will be considered together with an analysis of the effect of the scale setting procedure on the extracted values of $\Lambda_{\overline{M S}}^{\left(N_{\mathrm{f}}=2\right)}$.

\section{Acknowledgements}

We thank Michele Della Morte, Andreas Jüttner and Eigo Shintani for useful discussions. Our calculations were performed on the "Wilson" HPC Cluster at the Institute of Nuclear Physics, University of Mainz. We thank D. Djukanovic and C. Seiwerth for technical support. We are grateful for computer time allocated to project HMZ21 on the BlueGene computers JUGENE and JUQUEEN at NIC, Jülich. This research has been supported in part by the DFG in the SFB 1044 . We thank our colleagues from the CLS initiative for sharing the ensembles used in this work.

\section{References}

[1] M. Della Morte, B. Jäger, A. Jüttner and H. Wittig, JHEP 1203 (2012) 055 [arXiv:1112.2894].

[2] P. Fritzsch et al. [ALPHA Collaboration], Nucl. Phys. B 865 (2012) 397 [arXiv:1205.5380].

[3] K. G. Chetyrkin, J. H. Kühn and M. Steinhauser, Nucl. Phys. B 482 (1996) 213 [hep-ph/9606230].

[4] P. A. Baikov, K. G. Chetyrkin, J. H. Kühn and J. Rittinger, JHEP 1207 (2012) 017 [arXiv:1206.1284].

[5] K. G. Chetyrkin, V. P. Spiridonov and S. G. Gorishnii, Phys. Lett. B 160 (1985) 149.

[6] T. van Ritbergen, J. A. M. Vermaseren and S. A. Larin, Phys. Lett. B 400 (1997) 379 [hep-ph/9701390].

[7] K. G. Chetyrkin and A. Retey, Nucl. Phys. B 583 (2000) 3 [hep-ph/9910332].

[8] M. Göckeler et al. [QCDSF Collaboration], Nucl. Phys. B 688 (2004) 135 [hep-lat/0312032].

[9] E. Shintani et al. [JLQCD and TWQCD Collaborations], Phys. Rev. D 79 (2009) 074510 [arXiv:0807.0556].

[10] E. Shintani et al. [JLQCD Collaboration], Phys. Rev. D 82 (2010) 074505 [arXiv:1002.0371].

[11] S. Aoki et al. [FLAG Working Group], [arXiv:1310.8555].

[12] A. Francis, B. Jäger, H. B. Meyer and H. Wittig, Phys. Rev. D 88 (2013) 054502 [arXiv:1306.2532].

[13] H. Horch, M. Della Morte, G. Herdoíza, B. Jäger, A. Jüttner, H. Wittig, PoS LATTICE 2013 (2013) 304 [arXiv:1311.6975].

[14] A. Francis, B. Jäger, H. B. Meyer and H. Wittig, PoS LATTICE 2013 (2013) 305 [arXiv:1311.3285]. 